\title{
La simulación como herramienta de evaluación de competencias y certificación
}

\author{
Simulation as a tool of competence's evaluation and certification
}

Claudio Nazar J,* Nicole Bloch G, ${ }^{\ddagger}$ Ricardo Fuentes H*

Palabras clave:

Simulación, educación médica, evaluación, certificación, competencias.

Keywords: Simulation, medical education, assessment, certification, competence.

\section{Declaración de}

Conflicto de intereses: Los autores declaran no tener ningún conflicto de intereses.

* Profesor Asociado, División de

Anestesiología.

‡ Interno de Medicina.

Escuela de Medicina, Facultad de Medicina, Pontificia Universidad Católica de Chile.

\section{RESUMEN}

La simulación ha sido definida como la técnica de imitar la conducta, situación, procedimiento o proceso por medio de un aparato o escenario adecuado, con propósitos de enseñanza-aprendizaje o de entrenamiento personal. A través de los años, su uso en educación médica ha ido en aumento de forma progresiva. La simulación tiene un nicho bien establecido en el proceso de enseñanza-aprendizaje de alumnos de pregrado y postgrado de medicina, pero para la evaluación de competencias y certificación esta técnica aún se encuentra subutilizada, en especial en nuestro país. En países anglosajones como Estados Unidos, Australia y Canadá, tienen más experiencia en su uso como instrumento de evaluación y certificación de competencias, pero con escasas publicaciones al respecto. La certificación se define como una evaluación realizada al finalizar la formación del pregrado o postgrado con el objetivo de garantizar que se hayan adquirido los conocimientos, habilidades y aptitudes necesarios para ejercer de manera adecuada como profesionales médicos o especialistas. El desafío futuro en Chile es lograr integrar de manera estandarizada y validada la simulación en el proceso de evaluación, certificación y recertificación de competencias.

\section{ABSTRACT}

The simulation has been defined as the technique of imitating the behavior, situation, procedure or process using a suitable apparatus or setting, with the purpose of teach-learning and personal training. It's use in medical education has been progressively increasing over the years. The simulation has a well-established niche in teach-learning's process of under and graduate medical students; however, for evaluation of competences and certification is still underutilized, especially in our country. The Anglo-Saxon countries, such as United States, Australia and Canada, have more experience in its use as instrument of evaluation and certification of competences, but with little publications about this topic. Certification is defined as an evaluation performed at the end of under or graduate medical education training, in order to ensure the achievement of the knowledge, skills and attitudes needed to properly exercise as doctors and specialists. The future challenge in Chile is to integrate in a standardized and validated way the simulation in educational assessment, certification and recertification of competences.

\section{INTRODUCCIÓN}

L a simulación ha sido definida como: "la técnica de imitar una conducta, situación, procedimiento o proceso por medio de un aparato o escenario adecuado, con propósitos de estudio o de entrenamiento personal". 1

Durante la última década, la educación médica ha presentado un incremento significativo en el uso de esta herramienta como método de enseñanza-aprendizaje, y también con el fin de evaluar habilidades clínicas. ${ }^{2}$ Esto ha llevado a un cambio en el paradigma educacional tradicional, desde los clásicos métodos ilustrativos o clases magistrales a una enseñanza basada en la experiencia y el autoaprendizaje. ${ }^{3}$ La simulación permite un entorno seguro, donde se puede aprender de manera activa, a través del ensayo y error, con retroalimentación por parte de los educadores de forma inmediata (debriefing) y sin poner en riesgo la seguridad del paciente. 4

La enseñanza basada en la simulación ha demostrado ser superior a los métodos tradicionales. ${ }^{5}$ En educación de pregrado, se ha utilizado de manera efectiva para instruir en ciencias básicas, en particular en fisiología, con gran aceptación por parte de los estudiantes ${ }^{6}$ para practicar un adecuado examen físico, en el entrenamiento de habilidades quirúrgicas, ${ }^{7}$ 
entre otras. También se ha usado con éxito en distintas especialidades médicas como anestesiología, existiendo evidencia de su capacidad para aumentar las competencias del equipo anestésico tanto en procedimientos invasivos como en distintas situaciones clínicas, de forma especial en casos de urgencias y emergencias médicas. ${ }^{8}$ Además, se ha encontrado utilidad en otras especialidades como cirugía, ${ }^{9}$ obstetricia, ${ }^{10}$ medicina de urgencia, pediatría ${ }^{11}$ y cuidados intensivos. ${ }^{12}$

El uso de tecnologías de simulación y su utilidad está bien establecido en la enseñanza médica de alumnos de pregrado y postgrado; sin embargo, su uso para acreditación y certificación aún se encuentra poco documentado en la literatura médica.

En la actualidad, la educación médica se encuentra en un punto de inflexión, con un cambio de paradigma desde la enseñanza y aprendizaje basado en la ganancia de conocimientos teóricos de manera pasiva, hasta aquélla regida por la adquisición personal de un conjunto de competencias, lo que lleva a la necesidad de evaluación, certificación y educación continua a lo largo de la vida profesional. ${ }^{13}$

\section{METODOLOGÍAS DE EVALUACIÓN}

Las evaluaciones son parte esencial de todo aprendizaje y proceso de entrenamiento. Existen dos tipos: las formativas y las sumativas. Las primeras tienden a aumentar la motivación personal de los estudiantes y elevar sus propios estándares de conocimiento, reconociendo los aspectos a mejorar. ${ }^{14}$ Las segundas, tienen como finalidad regular y asegurar la adquisición

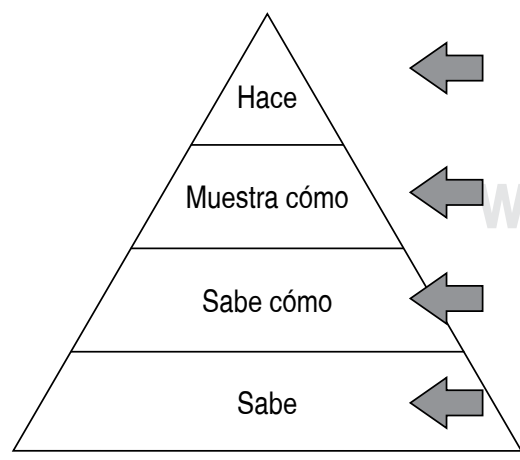

Observación del desempeño clínico: observación directa, videograbaciones, pacientes simulados incógnitos.

Pruebas de desempeño clínico: ECOE, simulaciones, pacientes estandarizados.

Aplicación del conocimiento: resolución de casos clínicos.

Evaluación del conocimiento: exámenes escritos, orales.

Figura 1: Pirámide de Miller e instrumentos de evaluación sugeridos para cada nivel. de la competencia profesional, dejando la necesidad de aprender en un segundo plano. ${ }^{15}$

En cuanto a las competencias necesarias en el ámbito de la Medicina, Miller establece un modelo en el que expone una pirámide con cuatro niveles y los instrumentos de evaluación sugeridos para cada nivel, como lo ilustra la Figura $1 .{ }^{16}$

- Primer nivel: saber. Se refiere al conocimiento teórico, evaluado de manera tradicional por pruebas escritas, en las cuales hoy predomina el formato de "selección múltiple". ${ }^{17}$

- Segundo nivel: saber cómo. Este nivel incluye la capacidad de resolver problemas o casos clínicos y describir procedimientos, evaluado de la manera clásica con exámenes orales o pruebas escritas.

- Tercer nivel: muestra cómo. Aquí el examinado debe demostrar sus competencias médicas. En el caso de varias escuelas de medicina chilenas, la Evaluación Clínica Objetiva Estructurada (ECOE) es utilizada de forma rutinaria desde hace ya varios años para evaluar este aspecto. ${ }^{18}$

La ECOE fue introducida por primera vez en 1975 por R. Harden, como un método de evaluación válido y fiable. ${ }^{19}$ Desde entonces ha sido aplicada para evaluar habilidades clínicas en escuelas de medicina y en exámenes internacionales de alto nivel. ${ }^{20}$ En ésta se incorporan diferentes métodos evaluativos, incluida la simulación que utiliza pacientes simulados o estandarizados, maniquíes de baja, media y alta fidelidad, interpretación de exámenes de laboratorio e imágenes, programas computacionales, entre otros. $^{21}$

- Cuarto nivel: hacer. Éste es el último nivel de la pirámide de Miller y corresponde a la capacidad del individuo para desenvolverse de manera adecuada en un escenario real de trabajo. Existen pocas estrategias validadas para evaluar este nivel, en este caso las más utilizadas son las pautas de observación directa, con la consecuente variabilidad y subjetividad interobservador. Como todo método de evaluación tiene sus limitaciones; la más significativa es que, al poseer cada 
evaluador sus propios estándares podría darse un sesgo por raza, sexo, entre otros elementos. ${ }^{22}$ Es factible que este nivel también sea medido con pacientes simulados (que por lo general son pacientes incógnitos, capaces de mantener el

Tabla 1: Dimensiones de la competencia profesional.

\section{Cognitiva}

Conocimiento básico

Capacidades básicas de comunicación

Manejo de información

Aplicar conocimiento a situaciones reales

Utilizar conocimiento y experiencias personales

Solución de problemas abstractos

Autogestión de adquisición de nuevos conocimientos

Reconocer brechas en el conocimiento

Generar preguntas

Uso de recursos

Aprender de la experiencia

\section{Técnica}

Habilidades en examen físico

Habilidades quirúrgicas o en realización de procedimientos

\section{Integrativa}

Integración de juicio científico, clínico y humanístico

Adecuado uso de estrategias de razonamiento clínico

Integrar conocimientos básicos y clínicos a través de disciplinas

Manejo de la incertidumbre

\section{Contexto}

Entorno clínico

Uso del tiempo

\section{Relacional}

Habilidades comunicativas

Manejo de conflictos

Trabajo en equipo

Enseñar a otros

\section{Afectivo/Moral}

Tolerancia a la ansiedad y ambigüedad

Inteligencia emocional

Respeto a los pacientes

Sensibilidad hacia los pacientes y la sociedad

Preocupación

\section{Hábitos mentales}

Observación de los propios pensamientos, emociones y técnicas

Atención

Curiosidad crítica

Reconocer los propios sesgos en conocimiento y emociones

Capacidad de reconocer y corregir errores "escenario real" durante el desempeño del alumno) y/o situaciones clínicas estandarizadas en las que, de nueva cuenta, la simulación ocupa un lugar en el sistema de evaluación. ${ }^{23}$

No existe metodología o sistema de evaluación perfectos, cada uno tiene sus fortalezas y debilidades; no obstante, el uso conjunto de varios podría compensar las carencias de cada método en particular. ${ }^{22}$ En el caso de la simulación como instrumento de evaluación, convendría usar diferentes técnicas para mejorar la confiabilidad del proceso evaluativo, por ejemplo, mezclar fantomas de alta fidelidad con pacientes estandarizados, creando un modelo híbrido, que permita valorar al mismo tiempo diferentes tipos de habilidades técnicas y no técnicas.

\section{LA SIMULACIÓN COMO HERRAMIENTA DE EVALUACIÓN Y MANTENCIÓN DE COMPETENCIAS}

Las instituciones de salud prestan servicios a la población, por lo que si se pretende otorgar servicios sanitarios de calidad se debe asegurar la competencia de sus profesionales.

El término competencia laboral es definido por la ley chilena como "todas las aptitudes, conocimientos y destrezas necesarias para cumplir exitosamente las actividades que componen una función laboral, según estándares definidos por el sector productivo" y evaluación de competencias laborales lo especifica como "un proceso de verificación del desempeño laboral de una persona contra una unidad de competencia laboral previamente acreditada" (Ley 20267, 2008). ${ }^{24}$ En el caso de los profesionales médicos, esta definición incorpora aspectos de competencias clínicas y relacionales, docentes, de investigación y de gestión. ${ }^{25}$ Es el uso habitual y juicioso de la comunicación, conocimientos, habilidades, razonamiento clínico, emociones, valores y reflexión en la práctica clínica diaria para el beneficio de los individuos y de la comunidad a la que se sirve. ${ }^{23}$ Las distintas dimensiones consideradas en la competencia clínica se describen en la Tabla 1.

En 1999, el Accreditation Council for Graduate Medical Education (ACGME) y el Ameri- 
can Board of Medical Specialties definió competencia médica con el cumplimiento de seis dominios: cuidado de pacientes, conocimiento médico, educación basada en la práctica, capacidades interpersonales y comunicacionales, profesionalismo y práctica sistemática. Estos requisitos comunes obligan a los programas de pregrado y residencia de medicina a educar en tales dominios y confirmar que sus alumnos sean competentes en cada uno de ellos. La simulación se incorporó de manera paulatina como metodología para valorar la adquisición de esta competencia médica, incluyendo pacientes estandarizados y simuladores mecánicos. Ésta resultó más efectiva para enseñar, aprender y evaluar algunas de las llamadas competencias no técnicas o "blandas", como las habilidades actitudinales, de comunicación y profesionalismo. ${ }^{26}$

Uno de los primeros usos de la simulación como herramienta para asegurar la calidad de un profesional es la certificación del Basic Life Support (BLS) y Advanced Cardiac Life Support (ACLS). El ACLS consta de un formato de aprendizaje en el cual los estudiantes revisan el material teórico en línea, y luego participan en escenarios clínicos simulados y estaciones de aprendizaje guiados por un instructor. ${ }^{27}$ Estos certificados por lo regular son exigidos al personal médico que trabaja en medicina de urgencia y cuidados intensivos y deben revalidarse cada dos años para mantener su vigencia. ${ }^{28}$ Además, está comprobado que la simulación para enseñar y aprender reanimación cardiopulmonar permite alcanzar con mayor rapidez la competencia así como a mantener niveles más altos de desempeño en residentes de medicina interna. ${ }^{29}$

Conocer el grado de dominio que una persona ha adquirido de una competencia es una tarea compleja y difícil. Implica partir con situaciones que simulen contextos clínicos reales lo más fidedigno posible y disponer de los medios de evaluación específicos para cada uno de los componentes de la competencia. Existen distintos estudios en los que se utiliza la simulación en la evaluación de competencias específicas del personal de salud de distintas especialidades, demostrando su utilidad y efectividad. Barsuk y colaboradores presentaron un sistema de trabajo para evaluar la habilidad de instalación de catéteres venosos centrales en fantomas. ${ }^{30}$ Young y su grupo pusieron a prueba a estudiantes de medicina y residentes de especialidades en el manejo de escenarios simulados de alto riesgo, demostrando diferencias significativas en el desempeño de los distintos niveles de alumnos, siendo éste mejor cuanto mayor es la experiencia de los estudiantes. ${ }^{31}$ Stefanidis y su equipo ${ }^{32}$ y Korndorffer y asociados ${ }^{33}$ demostraron que la simulación en cirugía laparoscópica fue eficaz en el entrenamiento y mantención de habilidades quirúrgicas, y mostraron mejoría significativa en el desempeño global en el pabellón de los residentes de cirugía.

\section{LA SIMULACIÓN PARA CERTIFICACIÓN Y MANTENCIÓN DE LA MISMA}

Se pueden distinguir al menos tres niveles diferentes de evaluación en el desarrollo profesional de un médico: ${ }^{21}$

1. Al terminar su formación en pregrado, con el propósito de certificar la adecuada adquisición de competencias conducentes al título de médico-cirujano.

2. Al terminar su formación de postgrado, con propósitos de certificación de la especialidad médica respectiva.

3. De manera periódica durante el trabajo independiente de los profesionales médicos, con el propósito de recertificación y mantención de sus competencias.

Dado que en Chile existe una gran heterogeneidad en la evaluación de los profesionales médicos al finalizar su formación de postgrado y durante el ejercicio profesional, se utiliza la experiencia internacional, con frecuencia representada por países anglosajones como Estados Unidos (EUA), Australia y Canadá, para entender los procesos relacionados con certificación y recertificación.

La certificación es la evaluación realizada al finalizar la formación de postgrado con el objetivo de garantizar que se hayan adquirido aquellos conocimientos, habilidades (técnicas y no técnicas) y actitudes que son parte esencial de una especialidad médica respectiva. 
Los métodos de evaluación se basan cada vez más en evaluaciones tipo ECOE, en los cuales la simulación tiene un papel cada vez más relevante, como lo hemos mencionado con anterioridad. Estas evaluaciones son realizadas por las sociedades científicas de las diferentes especialidades y subespecialidades, denominadas en inglés Boards; que son las responsables de garantizar las competencias de los médicos especialistas, previo a su ejercicio profesional.

Por otro lado, la recertificación se basa en evaluar las competencias básicas y comunes a todos los profesionales que ya ejercen una especialidad determinada, valorando de manera periódica las competencias esenciales de dicha especialidad. En la recertificación son utilizados métodos de evaluación como la ECOE (también con importante papel de la simulación), actividades formativas y pautas de observación directa de la práctica profesional con pacientes reales. ${ }^{21}$

Cada vez es más importante asegurar la calidad del servicio entregado a los pacientes, por lo que se ha convertido en un objetivo fundamental el detectar y remediar problemas que pudieran poner en riesgo la seguridad de los pacientes e interferir en una adecuada entrega de cuidados médicos. Esto generó cambios a nivel de la educación médica y de los métodos de evaluación con el fin de mejorar la capacidad de determinar el nivel real de habilidades, conocimientos y actitudes del profesional, por lo cual se incorporó la simulación como método de certificación de competencias profesionales. Un ejemplo de lo anterior es el examen de Licencia Médica de Estados Unidos (United States Medical Licensing Examination [USMLE]), que fue la primera organización evaluativa en integrar casos de simulación en su evaluación. En 2004 agregaron la evaluación de habilidades técnicas y no técnicas, utilizando pacientes simulados y situaciones estandarizadas. ${ }^{34}$

Distintas especialidades médicas han incorporado la simulación para su certificación. Por ejemplo, Medicina Familiar en EUA ${ }^{35}$ y Medicina Interna en Canadá. ${ }^{36}$ Asimismo, Anestesiología es una especialidad que ha incorporado con gran eficacia la simulación, debido a la necesidad del manejo de situaciones de crisis poco comunes en la clínica habitual, como hipertermia maligna, shock anafiláctico, manejo de vía aérea difícil, etcétera. El Board Estadounidense de Anestesiología requiere una educación médica basada en la simulación para completar la certificación. ${ }^{37}$ El Comité de Examinación de Anestesiología del Board Israelí reportó el uso de simulación como un elemento crucial para la certificación de anestesiólogos, creando cinco escenarios estandarizados para valorar manejo de trauma, Advanced Cardiac Life Support (ACLS), manejo de diferentes crisis en pabellón quirúrgico, ventilación mecánica y administración de anestesia regional. ${ }^{38}$

\section{LASIMULACIÓN COMO HERRAMIENTA DE EVALUACIÓN EN CHILE}

El desarrollo de la simulación en el proceso de enseñanza-aprendizaje y evaluación en medicina en Chile es una práctica que lleva pocos años. Comenzó en 2003 en la Escuela de Medicina de la Pontificia Universidad Católica de Chile, con una escuela de actores que simulaban patologías al ser interrogados por alumnos de pregrado de la carrera. Luego, en 2004 el Instituto Duoc UC creó el primer centro técnico de simulación en salud de Chile. ${ }^{39}$ En los años siguientes, algunas escuelas de educación superior comenzaron a incorporar simuladores en su actividad docente. Así, en 2008 la Escuela de Medicina de la Universidad de los Andes fue la primera institución que desarrolló un proyecto de integración de la simulación en el currículo académico del Internado de Cirugía.

En la actualidad son numerosas las escuelas y facultades de medicina chilenas que han incorporado la simulación de manera formal como método de enseñanza-aprendizaje y/o instrumento de evaluación a sus modelos docentes y malla curricular. Un ejemplo de lo anterior es la ECOE, que es utilizada como método de evaluación en distintos cursos de pregrado de Medicina de varias universidades chilenas, así como también el Examen Único Nacional de Conocimientos de Medicina (EUNACOM), donde varias estaciones presentan situaciones estandarizadas, fantomas y pacientes entrenados. ${ }^{40}$

EI EUNACOM es un examen teóricopráctico de Medicina General, aplicado en Chile desde el año 2009 en reemplazo del Examen Médico Nacional (EMN). Es exigido para acceder a cargos médicos dependientes del Ministerio de Salud y a programas de espe- 
cialización que son financiados por el Estado. Además, su aprobación por parte de médicos titulados en el extranjero les permite revalidar automáticamente su título para ejercer como médicos generales en Chile. Está compuesto por dos secciones, una parte teórica y una práctica. La sección práctica utiliza el formato de ECOE y consiste en una evaluación clínica en un entorno de atención médica real y/o simulada estandarizada, distribuida en cuatro etapas correspondientes a cada una de las grandes áreas de la medicina: Cirugía, GinecologíaObstetricia, Medicina Interna y Pediatría. ${ }^{40}$

\section{CONCLUSIÓN}

La implementación de la simulación como herramienta de evaluación de competencias y certificación en programas de pregrado y postgrado de medicina se encuentra aún en proceso de desarrollo, de forma específica en nuestro país, principalmente a través de su uso en los ECOE con pacientes estandarizados y fantomas de diferentes fidelidades. Las técnicas de simulación han repercutido de manera positiva en la educación médica moderna, en especial como herramienta de enseñanza-aprendizaje, pero todavía no han alcanzado su máximo potencial como instrumento de evaluación. El desafío futuro en Chile y en otros países de la región es lograr integrar a la simulación en procesos de evaluación, certificación y recertificación de competencias médicas, tal como lo han hecho algunos países anglosajones desde hace tiempo. Para lograr esta integración es necesario realizar estudios que logren estandarizar y validar su uso en estos ámbitos de la educación médica.

\section{REFERENCIAS}

1. Bradley P. The history of simulation in medical education and possible future directions. Med Educ. 2006; 40 (3): 254-262.

2. Scalese RJ, Obeso VT, Issenberg SB. Simulation technology for skills training and competency assessment in medical education. J Gen Intern Med. 2008; 23 (1 Suppl.): 46-49.

3. Clede L, Nazar C, Montaña R. Simulación en educación médica. Investig en Educ Médica [Internet]. 2014; 3 (10): 100-105. Disponible en http://linkinghub. elsevier.com/retrieve/pii/S2007505714727334

4. Rolfe I, Sanson-Fisher R. Professional development and ethics: translating learning principles into practice: a new strategy for learning clinical skills. Med Educ. 2002; 36 (4): 345-352.

5. Okuda Y, Bond W, Bonfante G, McLaughlin S, Spillane L, Wang E, et ál, National growth in simulation training within emergency medicine residency programs, 2003-2008. Acad Emerg Med. 2008; 15 (11): 11131116.

6. Via DK, Kyle RR, Trask JD, Shields CH, Mongan PD. Using high-fidelity patient simulation and an advanced distance education network to teach pharmacology to second-year medical students. J Clin Anesth. 2004; 16 (2): 144-151.

7. Lucas S, Tuncel A, Bensalah K, Zeltser I, Jenkins A, Pearle $M$, et ál, Virtual reality training improves simulated laparoscopic surgery performance in laparoscopy naïve medical students. J Endourol. 2008; 22 (5): 1047-1052.

8. Nishisaki A, Keren R, Nadkarni V. Does simulation improve patient safety?: self-efficacy, competence, operational performance, and patient safety. Anesthesiol Clin. 2007; 25 (2): 225-236.

9. Kneebone R. Simulation in surgical training: educational issues and practical implications. Med Educ [Internet]. 2003; 37 (3): 267-277. Available from: http://www. ncbi.nlm.nih.gov/pubmed/12603766

10. Dupuis O, Moreau R, Silveira R, Pham MT, Zentner A, Cucherat M, et ál, A new obstetric forceps for the training of junior doctors: a comparison of the spatial dispersion of forceps blade trajectories between junior and senior obstetricians. Am J Obstet Gynecol. 2006; 194 (6): 1524-1531.

11. Halamek LP, Kaegi DM, Gaba DM, Sowb YA, Smith BC, Smith BE, et ál, Time for a new paradigm in pediatric medical education: teaching neonatal resuscitation in a simulated delivery room environment. Pediatrics [Internet]. 2000; 106 (4): E45. Available from: http:// www.ncbi.nlm.nih.gov/pubmed/11015540

12. Hunt EA, Shilkofski NA, Stavroudis TA, Nelson KL. Simulation: translation to improved team performance. Anesthesiol Clin. 2007; 25 (2): 301-319.

13. Naik VN, Wong AK, Hamstra SJ. Review article: leading the future: guiding two predominant paradigm shifts in medical education through scholarship. Can J Anesth. 2012; 59 (2): 213-223.

14. Ben-David MF. The role of assessment in expanding professional horizons. Med Teach. 2002; 22 (5): 472-477.

15. Schuwirth $L$, van der Vleuten C. Merging views on assessment. Med Educ. 2004; 38 (12): 1208-1210.

16. Miller GE. The assessment of clinical skills/competence/ performance. Acad Med [Internet]. 1990 [citado $21 \mathrm{de}$ mayo de 2019]; 65 (9 Suppl): S63-S67. Disponible en http://www.ncbi.nlm.nih.gov/pubmed/2400509

17. Newble D. Techniques for measuring clinical competence: objective structured clinical examinations. Med Educ. 2004; 38 (2): 199-203.

18. Harden R, Stevenson M, Downie W, Wilson G. Assessment of clinical competence using objective structured examination. Br Med J. 1975; 1 (5955): 447-451.

19. Karam VY, Soo Park Y, Tekian A, Youssef N. Evaluating the validity evidence of an OSCE: results from a new medical school. BMC, Med Educ [Internet]. 2018 [citado 21 de mayo de 2019]; 18 (1): 313. Disponible en https://doi.org/10.1186/s12909-018-1421-x 
20. Daniels VJ, Pugh D. Twelve tips for developing an OSCE that measures what you want. Med Teach [Internet]. 2018; 40 (12): 1208-1213. Disponible en https://doi. org/10.1080/0142159X.2017.1390214

21. Martínez-Carretero J. Los métodos de evaluación de la competencia profesional: la evaluación clínica objetiva estructurada (ECOE). Educ Med [Internet]. 2005 [citado 21 de mayo de 2019]; 8 (Suppl 2): 1822. Disponible en http://scielo.isciii.es/pdf/edu/v8s2/ metodos.pdf

22. Epstein RM, Hundert EM. Defining and assessing professional competence. JAMA [Internet]. 2002 [citado 23 de mayo de 2019]; 287 (2): 226-235. Disponible en http://jama.jamanetwork.com/article. aspx?doi=10.1001/jama.287.2.226

23. Rethans JJ, Sturmans F, Drop R, Van Der Vleuten C. Assessment of the performance of general practitioners by the use of standardized (simulated) patients. $\mathrm{Br} J$ Gen Pract [Internet]. 1991 [citado 21 de mayo de 2019]; 41 (344): 97-99. Disponible en: https://www. ncbi.nlm.nih.gov/pmc/articles/PMC1371620/pdf/ brjgenprac00070-0011.pdf

24. Ley-20267 25-jun-2008 Ministerio del Trabajo y Previsión Social, Subsecretaría Del Trabajo -Ley ChileBiblioteca del Congreso Nacional [Internet]. 2008 [citado 23 de mayo de 2019]. Disponible en https:// www.leychile.cl/Navegar?idNorma $=272829$

25. Muñoz-Seco E. Evaluación de la competencia de los médicos de familia en la práctica clínica en situación real. Barcelona: Universitat Autónoma de Barcelona; 2016. p. 184

26. Okuda Y, Bryson EO, DeMaria S, Jacobson L, Quinones J, Shen B, et ál. The utility of simulation in medical education: what is the evidence? Mt Sinai J Med A J Transl Pers Med [Internet]. 2009 [citado 21 de mayo de 2019]; 76 (4): 330-343. Disponible en http://doi. wiley.com/10.1002/msj.20127

27. American Heart Association. ACLS Provider course [Internet]. 2019 [citado 23 de mayo de 2019]. Disponible en: https://international.heart.org/es/ourcourses/acls-provider-course

28. Steadman RH, Huang YM. Simulation for quality assurance in training, credentialing and maintenance of certification. Best Pract Res Clin Anaesthesiol [Internet]. 2012; 26 (1): 3-15. Disponible en http:// dx.doi.org/10.1016/j.bpa.2012.01.002.

29. Wayne DB, Siddall VJ, Butter J, Fudala MJ, Wade LD, Feinglass J, et ál. A longitudinal study of internal medicine residents' retention of advanced cardiac life support skills. Acad Med [Internet]. 2006 [citado 23 de mayo de 2019]; 81 (10 Suppl): S9-S12. Disponible en: http://www.ncbi.nlm.nih.gov/pubmed/17001145

30. Barsuk JH, McGaghie WC, Cohen ER, Balachandran JS, Wayne DB. Use of simulation-based mastery learning to improve the quality of central venous catheter placement in a medical intensive care unit. J Hosp Med. 2009; 4 (7): 397-403.
31. Young JS, DuBose JE, Hedrick TL, Conaway MR, Nolley B. The use of "war games" to evaluate performance of students and residents in basic clinical scenarios: $\mathrm{A}$ disturbing analysis. J Trauma. 2007; 63 (3): 556-564.

32. Stefanidis D, Korndorffer JR, Sierra R, Touchard C, Dunne JB, Scott DJ. Skill retention following proficiency-based laparoscopic simulator training. Surgery. 2005; 138 (2): 165-170.

33. Korndorffer JR, Dunne JB, Sierra R, Stefanidis $D$, Touchard CL, Scott DJ. Simulator training for laparoscopic suturing using performance goals translates to the operating room. J Am Coll Surg. 2005; 201 (1): 23-29.

34. Dillon GF, Boulet JR, Hawkins RE, Swanson DB. Simulations in the United States Medical Licensing Examination (USMLE). Qual Saf Health Care [Internet]. 2004 [citado 23 de mayo de 2019]; 13 (Suppl 1): i41-i45. Disponible en http://www.ncbi.nlm.nih.gov/ pubmed/15465954

35. Hagen MD, Sumner W, Roussel G, Rovinelli R, Xu J. Computer-based testing in family practice certification and recertification. J Am Board Fam Med. 2009; 16 (3): 227-232.

36. Hatala R, Barry K, Nishikawa. J Incorporating simulation technology in a Canadian internal medicine specialty examination: a descriptive report. Acad Med [Internet]. 2005 [citado 23 de mayo de 2019]; 80 (6): 554-556. Disponible en https://insights.ovid.com/ pubmed?pmid $=15917358$

37. The American Board of Anesthesiology. About MOCA 2.0 [Internet]. [citado 23 de mayo de 2019]. Disponible en http://www.theaba.org/MOCA/AboutMOCA-2-0

38. Berkenstadt H, Ziv A, Gafni N, Sidi A. Incorporating simulation-based objective structured clinical examination into the Israeli National Board examination in anesthesiology. Anesth Analg. 2006; 102 (3): 853-858.

39. Corvetto M, Bravo MP, Montaña R, Utili F, Escudero E, Boza C, et ál. Simulación en educación médica: Una sinopsis. Rev Med Chil. 2013; 141 (1): 70-79.

40. ASOFAMECH. Examen Único Nacional de Conocimientos de Medicina [Internet]. 2019 [citado 23 de mayo de 2019]. Disponible en http://www. eunacom.cl/organizacion/organizacion.html
Correspondencia:

Ricardo Fuentes $\mathbf{H}$

Marcoleta 377, 4o piso,

Edificio Carlos Casanueva, 6510260, Santiago, Chile.

Tel: 2639-8766 / 2354- 3270

Fax: 2632-7620

E-mail: rfuente@med.puc.cl 